\title{
The application of the Capon method to the parameter estimation of infrasound signals
}

\author{
Liang Meng, Xi-Hai Li, Wan-Gang Zhang, Dai-Zhi Liu \\ Xi'an Research Institute of High Technology, Xi'an 710025, People's Republic of China \\ ml7290@sohu.com
}

Keywords:nuclear tests; infrasound; Capon; azimuth;FK

\begin{abstract}
The Comprehensive Nuclear-Test-Ban Treaty adopted four technical means to monitor nuclear tests, which are the seismic, radionuclide, hydroacoustic and infrasound monitoring techniques. The infrasound monitoring technology is an effective tool for monitoring the atmosphere, ground or water nuclear explosions. The FK method is an array signal processing method used to estimate the slowness and azimuth of infrasound signals, but the resolution of this method is not high. This method is also susceptible to the influence of noise and the pseudo-peak is obvious. So this article introduces a High-resolution method-the Capon method, this method has been widely used in the signal processing of radar, communication and sonar. Based on the features and characteristics of the infrasound signals, the performance of the algorithm is analyzed and compared with the FK method, the results show that this method is superior to the FK method.
\end{abstract}

\section{Introduction}

On september 10, 1996, the Comprehensive Nuclear-Test-Ban Treaty (CTBT) was approved at the 50th session of the United Nations, the signing of the treaty in the field of arms control was significant[1]. In order to ensure the implementation of the treaty, the Comprehensive Nuclear-Test-Ban Treaty Organization (CTBTO)was established, and four techniques were used for monitoring nuclear tests, which are the seismic, radionuclide, hydroacoustic and infrasound monitoring[2]. The International Monitoring System (IMS) was also established in the worldwide. The infrasound monitoring technology was an effective method for monitoring the atmosphere, ground or water nuclear explosion[3]. Thus, the study of infrasound signals caused for great attention.

The FK method is an array signal processing method used to estimate the slowness and azimuth of signal. This method has been widely used in the analysis of seismic signal processing. Due to the similarity of the infrasound array and seismic array, the FK method has been extended to the analysis of infrasound signal processing, including the design of infrasound stations, the computation of slowness and azimuth[4]. Many researchers have studied on the method, such as the broadband FK method[5], the maximum likelihood estimation method[6]. The most commonly used method is the broadband FK method proposed by Kvaerna and Doorbvos[7]. But when the FK metod is applied to the infrasound signals, the resolution of slowness and azimuth is not high. This method is also susceptible to the influence of noise and the pseudo-peak is obvious. The accuracy of FK method is seriously affected .

Capon proposed the minimum variance beamforming methods, namely the Capon method[8], which marks in improving the resolution of an array antenna made pioneering achievements. Compared with conventional methods, Capon method provides a higher resolution. Currently, this method has been widely used in radar, communications, sonar and other fields[9,10], while in the research of infrasound, the high-resolution methods are used little. Therefore, according to the characteristic of infrasound signals, the Capon method is applied to the infrasound signals for high resolution to improve the accuracy of infrasound signal's azimuth. 


\section{The principle of capon algorithm}

The model of Array signals is as follows:

$$
X(t)=A\left(\theta_{0}\right) s_{0}(t)+N(t)
$$

Where $X(t)$ is the received data of array, $s_{0}(t)$ is the source signal received by the reference element, $A\left(\theta_{0}\right)$ is the array manifold, $N(t)$ is the noise signal.

In the beamforming, the weighting vector can recover the signal $s_{0}(t)$ from the fast shot $X(t)$ in the array:

$$
\begin{gathered}
s_{0}(t)=\langle W, X(t)\rangle=\left\langle W, A\left(\theta_{0}\right) s_{0}(t)+N(t)\right\rangle= \\
s_{0}(t) W^{H} A\left(\theta_{0}\right)+W^{H} N(t)
\end{gathered}
$$

The principle of Capon require to meet the signal variance unbiased estimator and the estimated minimum conditions, with the formulas are:

$$
\left\{\begin{array}{l}
W^{H} A\left(\theta_{0}\right)=1 \\
\min _{w} W^{H} R_{x} W
\end{array}\right.
$$

Where $R_{x}$ is the covariance matrix array. According to the Lagrange method, the optimal weight is get as:

$$
W_{\text {opt }}=\frac{R_{x}^{-1} A\left(\theta_{0}\right)}{A^{H}\left(\theta_{0}\right) R_{x}^{-1} A\left(\theta_{0}\right)}
$$

The output power of the array

$$
P\left(\theta_{0}\right)=W_{o p t}^{H} R_{x} W_{\text {opt }}=\frac{1}{A^{H}\left(\theta_{0}\right) R_{x}^{-1} A\left(\theta_{0}\right)}
$$

For the scanning of whole direction to obtain spectrum, the spectral peak corresponding to the wave direction is the azimuth of signal.

\section{The experiment}

Firstly the experiment uses the complex sinusoidal signal to analog the infrasound signal. Because the suspected nuclear frequency range of the IMS concern is generally $0.1-4 \mathrm{~Hz}$, the frequency of simulated signal is set to $2 \mathrm{~Hz}$. The infrasound signals of general monitoring are the far-field array signal. Assuming that the speed of infrasound signal is $340 \mathrm{~m} / \mathrm{s}$ and the azimuth is $45^{\circ}$ through the array, the array is a uniform linear array of four elements, spacing 85 meters. The distribution of the four elements is shown in Figure 1.

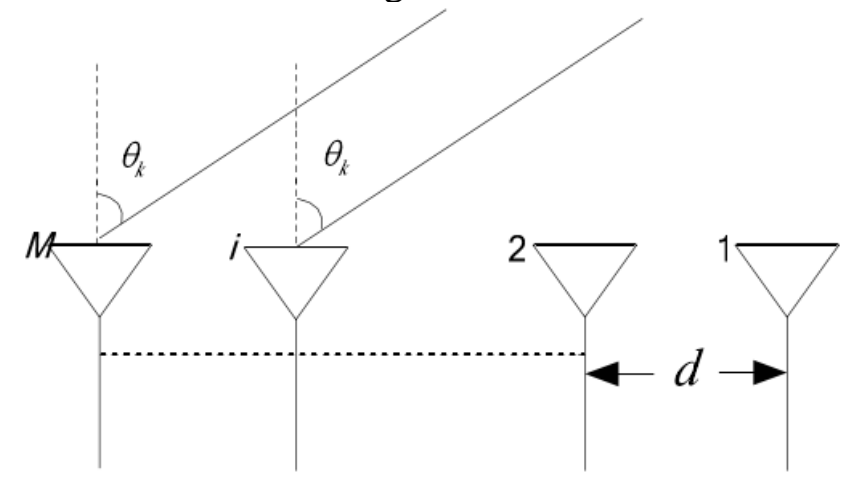

Fig.1 The signal model of the uniform linear array

For convenience of comparative analysis,the speed of sound is assumed to be a known quantity, the performance of the two algorithms to calculate azimuth is analyzed. At 10dB SNR conditions, the calculation result of the Capon method is shown in 2 (a); the spatial spectrum of the FK method with changes in azimuth is shown in 2 (b) below. As can be seen from the contrast figures, the result 
of the Capon method is significantly better than the FK method, the resolution of azimuth is higher.

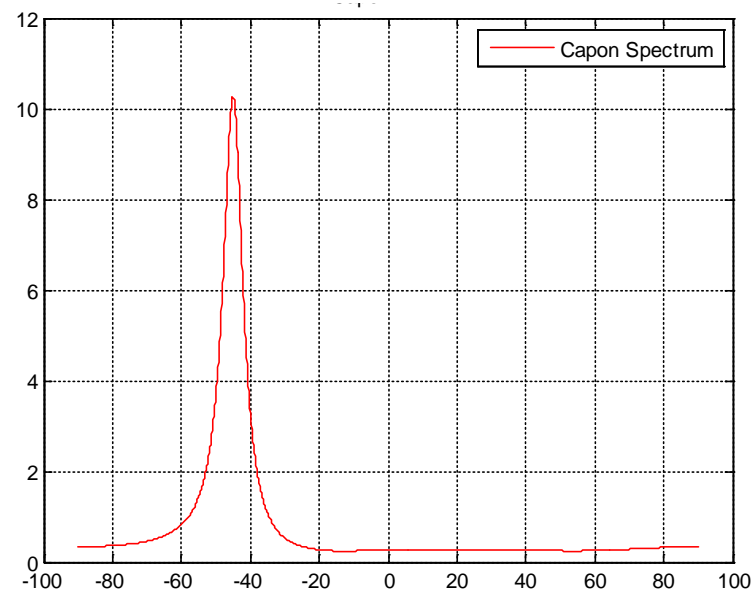

(a) the Capon method

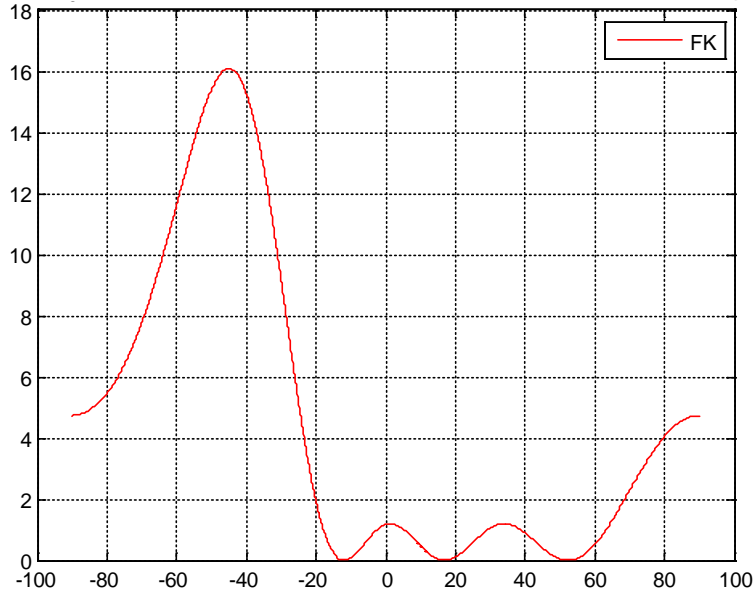

(b) the FK method

Fig.2 The spatial spectrum with changes in azimuth of the two algorithms

In order to further verify the effectiveness of the algorithm, the Gaussian modulated sine signal is used to analog the infrasound signal. the center frequency of the analog signal is $2 \mathrm{~Hz}$. The SNR is $10 \mathrm{~dB}$, the length of the analog signal are 2000 points, the sampling frequency is $20 \mathrm{~Hz}$. The analog signal is shown in Figure 3. The results of the two methods are shown in Figure 4, for the analog infrasound signal of the Gaussian modulated sine signal, the result of the Capon method is better than the FK method.

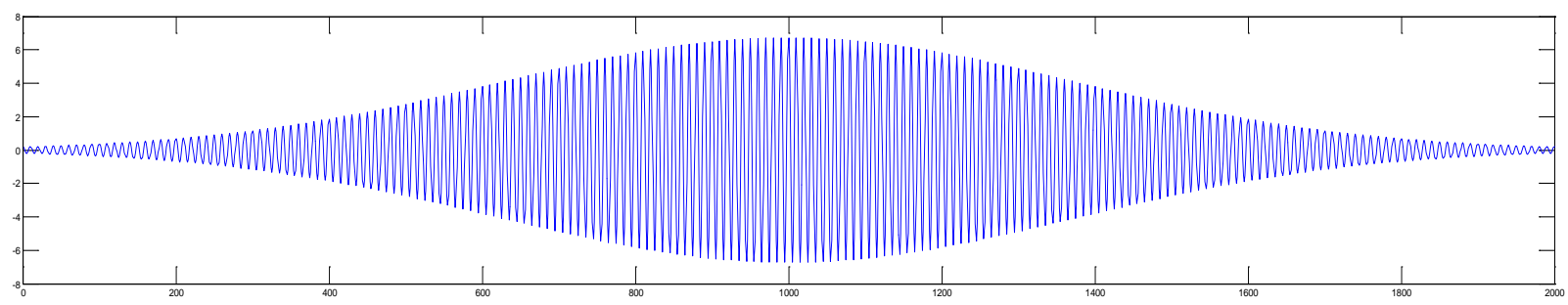

Fig.3 The analog infrasound signal of the Gaussian modulated sine signal
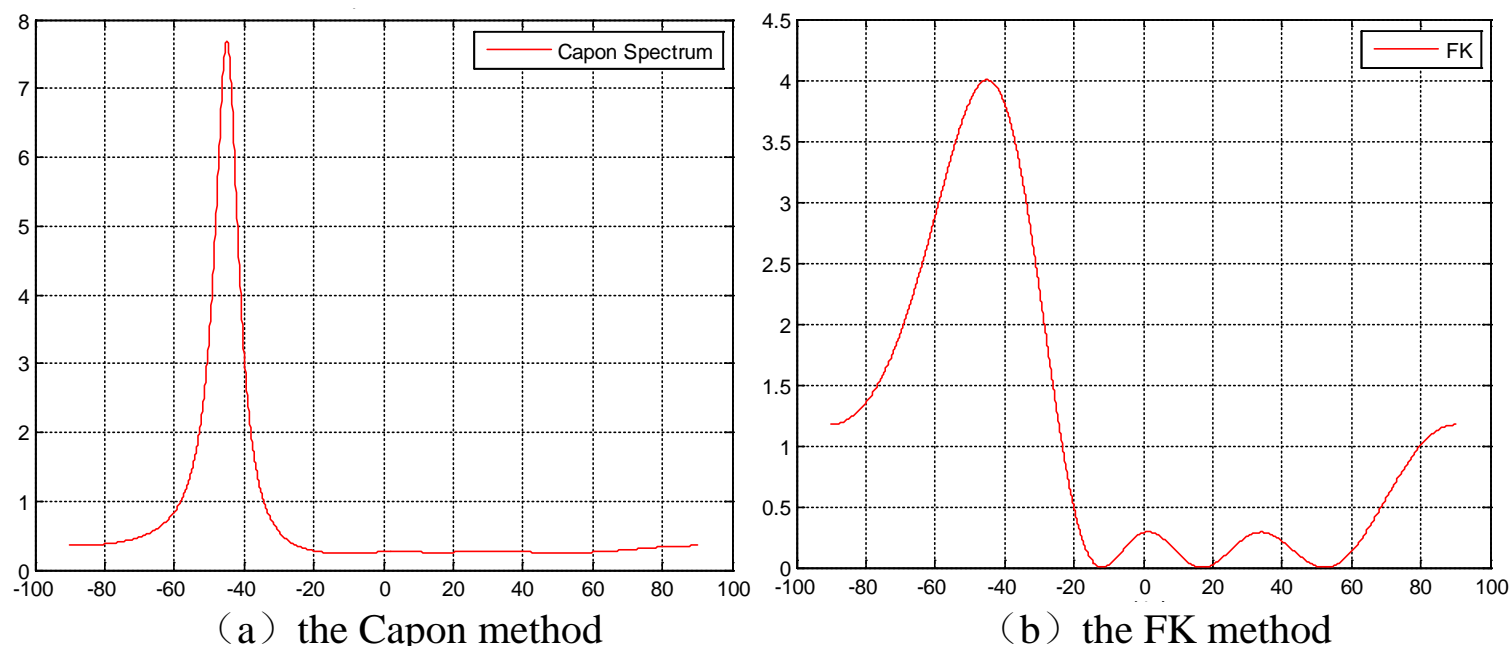

Fig.4 The spatial spectrum with changes in azimuth of the two algorithms

Finally, the actual infrasound array of IS55 is used to verify the algorithm. The CTBTO International Monitoring System's infrasound station IS55 is located at Windless Bight on the Ross Ice Shelf, Antarctica. The IS55 infrasonic array is an nine-element array divided into two sub-arrays. The element distribution of IS55 is shown in Figure 5. 


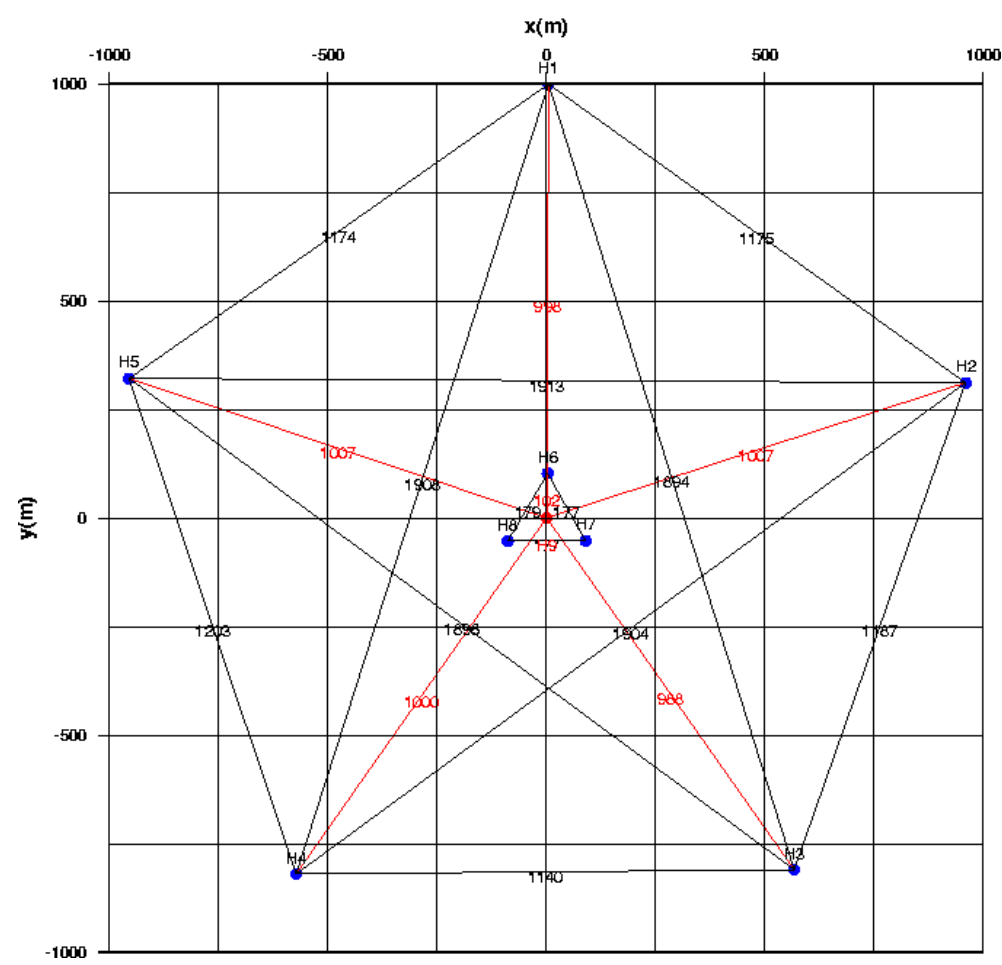

Fig.5 The elements distribution of infrasound stations 55

The analog signal is a complex sinusoidal signal, the frequency is $2 \mathrm{~Hz}$, and the Gaussian white noise is added to the signal for the noise ratio of $10 \mathrm{~dB}$. The results of the two methods shown in Figure 6. For the actual station, the Capon method is equally applicable, and the result is better than the FK method.
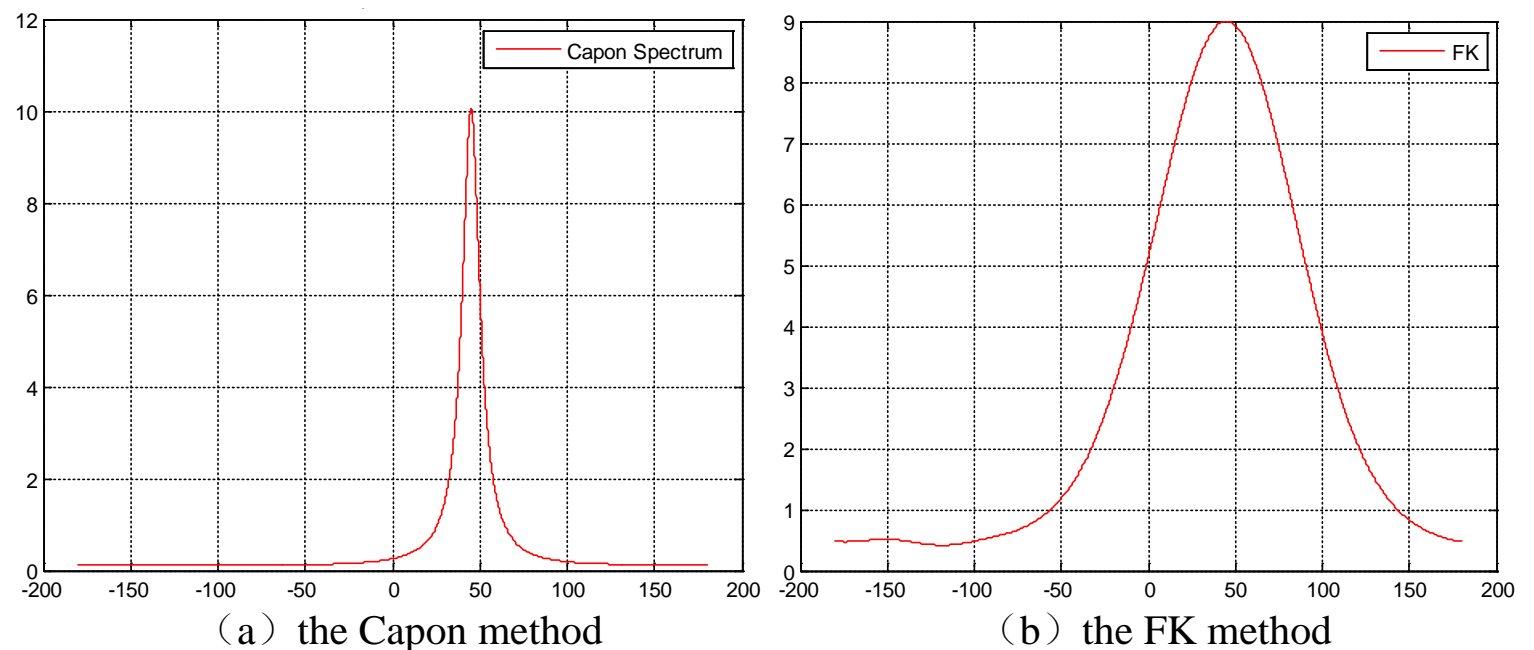

Fig.6 The spatial spectrum with changes in azimuth of the two algorithms

\section{Discussion and conclusions}

The FK method is an array signal processing method used to estimate the slowness and azimuth of signal. But when the FK metod is applied to the infrasound signals, the resolution is not high, the result is easily affected by noise, and the pseudo peak is apparent. So in this paper a high resolution method-the Capon method is introduced.

According to the characteristics of the infrasound signals, the performance of the Capon method is analyzed. Firstly under linear array, for a infrasound signal of single frequency sine signal the method is compared to the FK method and the results show that this method is effective to the infrasound array signal processing, then the method is extended to the Gaussian modulated 
sinusoidal infrasound signal. Finally, based on the actual infrasound array, the single-frequency sinusoidal infrasound signal is researched and the results show that the Capon method also can realize the high resolution of azimuth calculation. Thus, the Capon method is suitable for parameter estimation of infrasound signal, and can achieve high resolution of parameter estimation.

\section{References and links}

[1] LIN Y G, JIANG M, WANG X H, DONG J. Infrasound monitoring technique and its applications in CTBT verification[C]. National Security Geophysical Series (seven): Geophysics and nuclear detection, Xi'an map publishing press, 2011: 87-94.

[2] ZHANG L X. Introduction CTBT verification technologies[M]. Beijing: National defence industry press, 2005: 4-8.

[3] Liu J M, Tang W, Wang X M, Tang H Z. Analysis of mechanism to produce infrasound signals and its characteristics[J]. Environmental Engineering, 2010, 28(4):92-96

[4] Dong M R, Dong Y F, Xu X Z. Structure Design of Infrasonic Monitoring Arrays Based on F-K Analysis [J]. Journal of System Simulation, 2007， 19(19): 4557-4560.

[5] Kvaerna T, D J Doornbos. An integrated approach to slowness analysis with arrays and three-component array data[A].in: Semiannual Technical Summary, 1October 1985-31 March1986. Kjeller, Norway: Sci. Rept. No. 2-85/86, NORSAR,1986.

[6] Kvaerna T, F Ringdal. Stability of various F-K estimation techniques[A].in: Semiannual Technical Summary, 1 April1986-30 September 1986, Sci. Rept. No. 1-86/87, Kjeller, Norway: NORSAR, 1986. 29-40.

[7] Yan F, Jin P, Fan G C. Comparison between Two Estimation Methods of Azimuth, Slowness of Seismic Signal in Frequency and Time Domains on Seismic Array [J]. Northwestern Seismological Journal, 2006, 28(4): 327-330.

[8] Capon J. High-resolution frequency-wavenumber spectrum analysis[J]. Proc. IEEE 57, 1969, 1408-1418.

[9] Feng Y, Hou S, Chen Q, Ze J Z, Sun Y. Weak Signal DOA Time-Delay Joint Capon Estimation Method in Presence of Strong Signals[J]. Aerospace Shanghai,2014,31(2):8-12.

[10]Tan W Q, Hou Y G.Estimation of direction of source arrival based upon MUSIC and Capon[J]. Journal of Nanchang Institute of Technology,2008,27(1):20-23. 